\title{
Production of ring-like structure in the cocoon of Hercules A
}

\author{
Curtis J. Saxton ${ }^{1,2}$ \\ and \\ Geoffrey V. Bicknell ${ }^{2,1}$ \\ and \\ Ralph S. Sutherland ${ }^{2}$
}

\begin{abstract}
The radio lobes of the radio galaxy Hercules A contain intriguing ring-like structures concentric with the jet axis. To investigate the occurrence of such features, we have used hydrodynamic simulations of jets with a range of Mach numbers (from $M=2$ to 50 ) and densities (down to a ratio of $10^{-4}$ relative to the background) to generate ray-traced images simulating synchrotron emission from the time-dependent shock structures. We compare these images with observations of Hercules A, and consider the physical nature and temporal evolution of the most plausible configurations. We find that the observed ring-like structures are well explained as nearly annular shocks propagating in the backflow surrounding the jet. We infer that the jet is oriented at between $30^{\circ}$ and $70^{\circ}$ to the line of sight, consistent with radio depolarization observations of Gizani \& Leahy. The observational lack of hot-spots at the extremities of the radio lobes, and the possible presence of a buried hot-spot near the base of the western lobe, are explained in terms of the intrinsic brightness fluctuations and dynamics of the terminal shock of an ultra-light, low Mach number jet that surges along its axis due to intermittent pinching and obstruction by turbulent backflow in the cocoon. We conclude from the appearance of both sides of the Hercules A, that both jets are on the verge of becoming fully turbulent.
\end{abstract}

Subject headings: hydrodynamics - galaxies: active - galaxies: individual (Hercules A; 3C 348) — galaxies: jets — radio continuum: galaxies — X-rays: galaxies

\footnotetext{
${ }^{1}$ Department of Physics \& Theoretical Physics, Faculty of Science, Australian National University, ACT 0200, Australia

${ }^{2}$ Research School of Astronomy \& Astrophysics, Mt Stromlo Observatory, Australian National University, Cotter Road, Weston ACT 2611, Australia
} 


\section{Introduction}

Hercules A (3C 348) is a luminous radio galaxy (Dreher \& Feigelson 1984; Mason et al. 1988 ) at a redshift of $z=0.154$, in a cluster of uncertain richness (Greenstein 1962; Owen \& Laing 1989; Yates et al. 1989; Allington-Smith et al. 1993). Its power density at $5 \mathrm{GHz}$, $P_{5 \mathrm{GHz}} \approx 6.9 \times 10^{25} \mathrm{~W} \mathrm{~Hz}^{-1} \mathrm{sr}^{-1}$ for $H_{0}=65 \mathrm{~km} \mathrm{~s}^{-1} \mathrm{Mpc}^{-1}$ (Gizani \& Leahy 1999; Gizani et al. 2000). In optical observations, the elliptical galaxy is found to have a double nucleus (with a separation $\sim 4^{\prime \prime}$ ) and tail-like distortions of the stellar isophotes implying a merger of galaxies (Minkowski 1957; Sadun \& Hayes 1993).

The X-ray emitting cluster medium was the subject of ASCA and ROSAT observations by Siebert et al. (1999) and ROSAT observations by Gizani \& Leahy (1999). The diffuse Xray distribution is elongated in the direction of the radio source axis, suggesting an interaction with the radio lobes. The medium is otherwise well described by a $\beta$-model profile with bestfit parameters $\beta \approx 0.63$ and core radius $r_{\mathrm{c}} \approx 35 \pm 3^{\prime \prime}$ (Siebert et al. 1999). The contribution from the nucleus is $\lesssim 3 \%$ of total X-ray luminosity, but the cluster surface brightness may be centrally peaked (Gizani \& Leahy 1999). However there may actually be two thermal X-ray emitting components present, as the ASCA spectrum suggests a temperature $k T \approx 4.3 \mathrm{keV}$ and a central electron density of $n_{0} \approx 9.1 \times 10^{-3} \mathrm{~cm}^{-3}$ (Siebert et al. 1999), whereas the ROSAT/PSPC spectrum suggests $k T \approx 2.4 \mathrm{keV}$. ROSAT observations by Gizani et al. (2002) determined a temperature $k T=2.45 \mathrm{keV}$ and $n_{0}=7.8 \times 10^{-3} \mathrm{~cm}^{-3}$. Trussoni et al. (2001) presented BeppoSAX observations indicating a temperature $4-5 \mathrm{keV}$ on scales of several arcminutes with the possibility of an additional $\sim 3 \mathrm{keV}$ component.

In VLA observations, Gizani \& Leahy (1999) found that the cluster medium affects the depolarization of the radio features: the western side exhibits greater depolarization than the east. As the more distant jet and lobe are seen through a thicker column of the cluster medium, they deduced a jet inclination of $\theta \approx 50^{\circ}$ to the line of sight. Thus Hercules $\mathrm{A}$ exhibits a classical Laing-Garrington effect (Laing 1988; Garrington et al. 1988).

The radio lobes span $\sim 3^{\prime}$ and in overall shape they are symmetric about the nucleus (see the upper panel of Figure 1). However, the eastern lobe accounts for $3 / 4$ of the total radio luminosity. Although the radio power of Hercules A is in the range of FR 2 sources (See Fanaroff \& Riley 1974) the morphology of the radio lobes belongs ambiguously to the FR 1 class. Most notably, neither lobe shows a hot-spot near its outer extremity.

The western radio lobe (on side of the receding jet) contains a sequence of arc-like and ring-like features that are brighter than the lobe, and which are approximately concentric

with the jet axis (Dreher \& Feigelson 1984; Mason et al. 1988). Some of the ring-like features are partially filled; some are open; some are incomplete. The most distinct of these features, 
and the furthest from the nucleus, is a "semi-ring" that is bright on only one side. Radio emission from the rings is linearly polarised: up to $\sim 50 \%$ in the two outer rings (Dreher \& Feigelson 1984). The radio spectral indices are flatter in the jets and rings $(\alpha \approx 1.1$ where $\left.S_{\nu} \propto \nu^{-\alpha}\right)$ than in the radio lobes generally $(\alpha \approx 1.6)$, indicating that the radio emitting plasma in the rings is younger than the background (Gizani \& Leahy 1999; Gizani et al. 2002). The eastern (approaching) jet shows "helical" features, which may be a projected superposition of rings (Gizani et al. 2002).

The radio lobes of some other galaxies display ring-like internal structures, which have been likened to the rings in the western lobe of Hercules A (Morrison \& Sadun 1996). The source 3C 310 also shows several circular features in both the northern and southern lobes; these rings are larger in proportion to the lobes than those of Hercules A (van Breugel \& Fomalont 1984; Morrison \& Sadun 1996). Other radio galaxies with ring-like features include MG $0248+0641$ (Conner et al. 1998) and 3C 219 (Perley et al. 1980).

Several explanations have been offered for the nature and origin of the rings. Dreher \& Feigelson (1984) suggested that the rings in Hercules A originate as distinct ejections from the nucleus, or interruptions of the jet due to either intrinsic pinching instabilities or collisions with clouds straying into the jet axis. In that case, the bright rings are surfaces where the ambient radio lobe or cluster medium contacts and interacts with a cloud.

Mason et al. (1988) proposed that the jet loses collimation at the point where the radio structure widens, and that concentrated streams of relativistic protons intermittently cross or self-cross, causing energetic particle bursts which deposit kinetic energy in the plasma at that site. These disturbances expand spherically, as they drift outwardly within the medium of the radio lobe. In this scenario, the rings are the projected edges of the spheres. This would account for the approximately linear relation between ring radius and displacement from the core.

Morrison \& Sadun (1996) proposed a qualitatively similar theory in which uniformly expanding spheres originate as acoustic disturbances carried outwardly in a galactic wind. The subsequent onset of jet activity then floods the region with radio-emitting material. Radio-bright plasma interacts with the shells and tends to concentrate on the surfaces of these structures. One implication of this model is that the extensive wind has an age of Gigayears. However, this requirement would be difficult to reconcile with the likely disturbances in the cluster environment of Hercules A, especially related to the merger of another galaxy with Hercules A (as implied by the double nucleus and stellar tail, Sadun \& Hayes 1993). Thus, models for the radio structure of Hercules $\mathrm{A}$ in which the formation of rings is intrinsic to the jet and lobe dynamics seem to have more appeal. 
Meier et al. (1991) proposed such a model, based on extensive numerical simulations, in which an over-dense jet undergoes a sudden expansion when it becomes highly overpressured some $20^{\prime \prime}$ from the core and subsequently expands conically giving rise to the "ice-cream cone" shape of the lobe. Meier et al. (1991) offered a qualitative explanation for the prominent rings in the western lobe as being the result of weak spherical shocks. Our difficulty with this model, which is really a difficulty with all models involving dense jets, is that the implied jet energy flux under most circumstances is too large. This is most likely the case for Hercules A. Moreover, the implied Mach number is also very large. In the next section, therefore, we examine the case for $\eta \sim 1$ in some detail and in so doing we present our case for a more conventional range of $\eta \ll 1$.

\section{Choice of parameters}

Useful constraints on the parameters of jets may be obtained by relating the energy flux to the observed radio power. The energy flux of a relativistic jet consisting of cold matter plus relativistic particles is given by the following: Let $\beta_{\text {jet }}=v_{\text {jet }} / c, \Gamma$ be the jet Lorentz factor, $p_{\text {jet }}$ be the jet pressure, $\chi=\rho c^{2} / 4 p_{\text {jet }}$ where $\rho_{\text {jet }}$ is the cold matter rest-mass density and $A_{\text {jet }}=\pi D_{\text {jet }}^{2} / 4$ be the jet cross-sectional area for diameter $D_{\text {jet }}$. Then, the energy flux, $F_{E}$ is given by:

$$
F_{E}=4 p_{\text {jet }} c \beta_{\text {jet }} A_{\text {jet }} \Gamma^{2}\left[1+\frac{\Gamma-1}{\Gamma} \chi\right]
$$

Bicknell (1994). The parameter, $\chi$ can be expressed in terms of $\eta$, the ratio of jet to ISM pressures and the ISM temperature, $T_{\text {ism }}=10^{7} T_{7} \mathrm{~K}$ as

$$
\chi=\eta\left(\frac{p_{\text {ism }}}{p_{\text {jet }}}\right)\left(\frac{\mu m_{p} c^{2}}{k T_{\text {ism }}}\right) \approx 1.7 \times 10^{5}\left(\frac{p_{\text {ism }}}{p_{\text {jet }}}\right) \eta T_{7}^{-1}
$$

Numerically, the energy flux is

$$
\begin{aligned}
F_{E} & \approx 9.0 \times 10^{42}\left(\frac{p_{\text {ism }}}{10^{-11} \mathrm{dyn} \mathrm{cm}^{-2}}\right)\left(\frac{D_{\text {jet }}}{\mathrm{kpc}}\right)^{2} \Gamma^{2} \beta_{\text {jet }} \\
& \times\left[\frac{p_{\text {jet }}}{p_{\text {ism }}}+1.7 \times 10^{5} \frac{\Gamma-1}{\Gamma} \eta T_{7}^{-1}\right] \operatorname{erg~s}^{-1}
\end{aligned}
$$

We have chosen a fiducial value of $1 \mathrm{kpc}$ for $D_{\text {jet }}$ since the $0.5^{\prime \prime}$ resolution limit of the observations (e.g. Dreher \& Feigelson 1984) implies an upper limit on the unresolved jet diameter of about $1.3 \mathrm{kpc}$. On the other hand the jet diameter at $20^{\prime \prime}$ is probably not much less than $0.5^{\prime \prime}$ since that corresponds to an opening angle of $0.6^{\circ}$. In the non-relativistic 
limit, for a hypersonic jet,

$$
F_{E} \approx \frac{1}{2} \rho_{\text {jet }} v_{\text {jet }}^{3} A_{\text {jet }} \approx 1.1 \times 10^{48} \eta\left(\frac{n_{\text {ism }}}{10^{-2} \mathrm{~cm}^{-3}}\right) \beta_{\text {jet }}^{3}\left(\frac{D_{\text {jet }}}{\mathrm{kpc}}\right)^{2} \mathrm{erg} \mathrm{s}^{-1}
$$

and this expression serves as a reasonable order of magnitude estimate of the effects of various parameters. We should compare these expressions for the jet energy flux to estimates of this parameter obtained from the radio power.

In making these comparisons, first note that the ISM parameters obtained from Xray observations summarised in the introduction, are reasonably well determined. The central electron number density density, $n_{e}$, of the interstellar medium is approximately $7.8 \times 10^{-3} \mathrm{~cm}^{-3}$ with a corresponding total number density, $n_{\text {ism }} \approx 1.5 \times 10^{-2} \mathrm{~cm}^{-3}$; the temperature is $2.45 \mathrm{keV} \approx 2.8 \times 10^{7} \mathrm{~K}$; the corresponding pressure, $p_{\text {ism }} \approx 5.7 \times 10^{-11} \mathrm{dyn} \mathrm{cm}^{-2}$. We use these central estimates of density and pressure within the core radius $\approx 35^{\prime \prime}$ of the hot gas distribution. Second, although we have allowed for a difference between jet and ISM pressure, we evaluate the above expressions for energy flux in the region within $20^{\prime \prime}$ of the core where both Meier et al. and we assume that the jet is confined. The jet is unresolved in this region so that we only know that $D \lesssim 500 \mathrm{pc}$; we have taken a fiducial value of $100 \mathrm{pc}$. Third, given that we are dealing with a supersonic jet, it is highly likely that the jet is at least mildly relativistic for the following reason. There currently seems to be little doubt that jets in radio loud objects are initially relativistic. Given that this is the case, a jet that is supersonic on the kpc scale has a velocity of at least $0.3 \mathrm{c}$ (Bicknell 1994). Using the flux density given in Kellermann et al. (1969), the total power of Hercules A at $1.4 \mathrm{GHz}$ is approximately $3.1 \times 10^{27} \mathrm{~W} \mathrm{~Hz}^{-1}$. For reasonable limits on the ratio of radio power to jet energy flux, $\kappa_{1.4} \sim 10^{-12}-10^{-11} \mathrm{~Hz}^{-1}$ (Bicknell 2000), the jet power is between $2 \times 10^{45}$ and $2 \times 10^{46} \mathrm{erg} \mathrm{s}^{-1}$. For $\beta=0.5$, and $\eta=1$, the energy flux given by equation (3) is approximately $3 \times 10^{47} \mathrm{erg} \mathrm{s}^{-1}$, about an order of magnitude too large.

Another problem with $\eta \sim 1$ is that the implied Mach number is enormous. For example, in the non-relativistic approximation, the jet Mach number, $M_{\text {jet }} \approx 710 \beta_{\text {jet }} T_{7}^{-1 / 2} \eta^{1 / 2}$ and the Mach number of 210 implied by $\beta_{\text {jet }} \sim 0.5$, and $\eta \sim 1$ is much larger than the value of 6 preferred by (Meier et al. 1991) in their explanation for the rings. Moreover, simulations with such a high Mach number are likely to be quite different from the ones presented by Meier et al. We expect that the jet and its cocoon would be much more needle-like in this case.

Another useful way to constrain the parameters of the jets in Hercules A is to express the energy flux in terms of $\eta$ and $M_{\text {jet }}$. For a hypersonic, non-relativistic jet

$$
F_{E} \approx \frac{1}{2} \rho_{\text {ism }}\left(\frac{p_{\text {jet }}}{p_{\text {ism }}}\right)^{3 / 2}\left(\frac{4 k T_{\text {ism }}}{3 \mu m_{p}}\right)^{3 / 2} \eta^{-1 / 2} M^{3} A_{\text {jet }}
$$




$$
\approx 2.9 \times 10^{44}\left(\frac{p_{\text {jet }}}{p_{\text {ism }}}\right)^{3 / 2}\left(\frac{n_{\text {ism }}}{10^{-2} \mathrm{~cm}^{-3}}\right)\left(\frac{\eta}{10^{-4}}\right)^{-1 / 2} T_{7}^{3 / 2}\left(\frac{M}{10}\right)^{3}\left(\frac{D}{\mathrm{kpc}}\right)^{2} \operatorname{erg~s}^{-1}(5)
$$

where $T_{7} \approx 2.8$ in Hercules $\mathrm{A}$. As we have seen above, and this expression confirms it, a value of $\eta \sim 1$ requires a Mach number $\sim 70-150$ to attain an energy flux $\sim 2 \times$ $10^{45-46} \mathrm{erg} \mathrm{s}^{-1}$. Again, these values considerably exceed the value of 6 favored by Meier et al. (1991). Conventional values of $\eta \ll 1$ are able to provide the requisite energy flux for more modest Mach numbers. For example, $\eta \sim 10^{-4}$ and $M \sim 10-12.5$ gives an energy flux of $2 \times 10^{45-46} \mathrm{erg} \mathrm{s}^{-1}$. For these reasons, therefore, we have restricted our simulations to (fairly wide) ranges in Mach number of $2-50$ and density ratio of $10^{-4}-10^{-2}$. As we shall see, there is some indication that the Mach number may be close to transonic and that correspondingly, the density ratio may be even lower than $10^{-4}$. However, we have not carried out simulations for $\eta<10^{-4}$ since computationally, these are prohibitively expensive as a result of the Courant condition.

An appealing aspect of the Meier et al. (1991) simulations is that, at approximately $20^{\prime \prime}$ from the core, the western jet becomes over-pressured. Indeed, inspection of the most recent image Gizani et al. (2000) shows that the western jet may be behaving like a classical over-pressured jet over at least part of the region between $20^{\prime \prime}$ and $60^{\prime \prime}$. The knots of emission in this region could well be the result of particle acceleration at reconfinement shocks. The characteristic expansion and compression of the jet is especially manifest in the lower panel of Figure 1 where the brightness and contrast of the Gizani et al. (2000) image has been adjusted to highlight faint features. Reconfinement features are also present in our simulations since the production of periodic shocks is relatively easy in a supersonic jet. The X-ray data (e.g. Siebert et al. 1999) provide a natural explanation for the jet becoming over-pressured in this region since the core radius is approximately $35^{\prime \prime}$ and $20^{\prime \prime}$ deprojected is approximately $26^{\prime \prime}$. At this point the background confining pressure would be starting to decrease from the central value. However, there is nothing in the X-ray data to suggest that the jet is becoming extremely overpressured at this point as required by the Meier et al. (1991) models.

Thus, for the above reasons, we have opted for a model for Hercules A in which the jet density ratio is much less than unity, the Mach number is not excessively large and the jet is initially confined. In Saxton et al. (2002) we simulated the interactions of a hypersonic, low density jet with its turbulent cocoon, covering a parameter-space with two orders of magnitude in jet density and Mach numbers from 5 to 50 . We considered the dynamics of point-like and ring-like shocks near the head of the jet, in order to model and interpret the complex shock structures observed near the western hot-spot of Pictor A. In the present paper, we use this simulation database, augmented by some additional simulations, to generate synthetic radio maps resembling the morphology and brightness distribution of the features of Hercules A. Our model for some of the observed radio rings is that they are 
open or partially filled annular shocks propagating in the cocoon backflow in the western radio lobe. It is feasible that other features that have been described as rings are in fact either related to jet reconfinement or recessed hot-spots in the lobe. At this stage we have not attempted to model the effects of reconfinement shocks, and we have concentrated on the production of rings near the head of the lobe. Therefore, we assume a constant background density in our simulations.

It is useful at this stage to refer to the lower panel of Figure 1 where we have labeled some of the regions in the western lobe according to our model and have shown the effects of ellipse fitting to the features that could be interpreted as rings. Note that these fits are not tightly constrained and depend to some extent upon what parts of the radio surface brightness are deemed to belong to a particular ring. We discuss the elliptical rings further in $\S 5.3$ with respect to the orientation of the jets.

\section{Simulations}

The hydrodynamic simulations were conducted using the Piecewise Parabolic Method (PPM) (Colella \& Woodward 1984) implemented in a code based on the VH-1 code (Blondin \& Lufkin 1993). An advantage of PPM for this type of simulation is its excellent shockcapturing properties. We have enhanced the code to achieve greater efficiency, have included code to correct for a numerical shock instability and have added a scalar tracer variable $\varphi$ that is passively advected with the flow. By assigning $\varphi=1$ within jet plasma entering the grid, and initially $\varphi=0$ in the background medium, we use this variable to distinguish and follow the evolution of the constituents of the physical system.

Our images of simulated radio surface brightness were rendered by a ray-tracing program that projects three-dimensional structures obtained from the PPM output. Assuming that the magnetic pressure is approximately proportional to the hydrodynamic pressure, $p$, the

volumetric emissivity, $j_{\nu} \propto \varphi p^{(3+\alpha) / 2}$. This ansatz is only valid at a frequency that is less than any cooling-induced break in frequency so that to be consistent we choose a value of $\alpha=0.6$. This is flatter than the observed spectral index in, for example, the rings wherein $\alpha=1.1$ (Gizani et al. 2002) indicating that these features are older than the synchrotron cooling time. Our assumption of $\alpha=0.6$ means that the relative contrast of features will be affected. However, the resulting images obtained by integrating $j_{\nu}$ along given lines of sight for each pixel of the simulated intensity map, should give a good qualitative indication of the appearance of the flow and also give a good indication of the relative contrast of features as a function of jet parameters. (This is an important point that is discussed below in $\S 5.2$.) 
Despite the importance of synchrotron and/or inverse Compton cooling for the radio spectrum, cooling does not affect the hydrodynamics of the flow since the lowest energy particles that dominate the internal energy are unaffected. Therefore, the way the system behaves depends on dimensionless parameters such as the density ratio of jet to ambient gas, $\eta$, the corresponding jet to ambient pressure ratio (assumed equal to one), and the Mach number of the jet, $M \equiv \eta^{-1 / 2} v_{\text {jet }} / c_{\mathrm{s} \text {,ism }}$ where $c_{\mathrm{s}, \text { ism }}$ is the sound speed in the interstellar medium. Our simulations cover a parameter space defined by $\eta=10^{-2}, 10^{-3}, 10^{-4}$ and $M=2,5,10,50$. Adopting the cooler estimate of the temperature and density in the ambient cluster medium as fiducial scales for our simulations, the thrust and power of the simulated jets are given in Table 1 . These are classical values, and can be related to parameters of relativistic jets with equivalent thrust or power (e.g. Rosen et al. 1999; Carvalho \& O'Dea 2001). The time interval between output frames of the hydrodynamic data was set so that approximately 600 frames were generated before the jet reached the right side (large $z$ ) boundary of the grid.

We assume that the outer boundaries of the computational grid are open to outflow, except at the base of the jet at the left (low- $z$ ) boundary. The initial properties of the jet are copied to those cells at the start of each time step, in order to maintain a jet with constant mass flux, thrust and power. In Saxton et al. (2002) we concentrated on cases in which a reflecting condition applies at the left boundary, representing effects near the plane of symmetry near the nucleus producing equally strong, opposite jets. The closed boundary condition was found to lead to a conical cocoon which is typically much wider than the cylindrical cocoons that develop about jets in simulations with an open left boundary. The latter represent the effects of systems where the head of the jet is causally distant from the nucleus and the plane of symmetry about the galaxy. We will initially focus our discussion on simulations with this type of boundary condition, as this seems physically appropriate for Hercules A, since the radio lobes extend hundreds of kiloparsecs from the galaxy.

\section{Results}

\subsection{Morphology}

The morphology of our simulated brightness maps is determined by the distribution of bright shock features. The termination shock of the jet is often, but not always, the brightest of such features. Biconical ("diamond") shocks also occur within the jet, initiated by disturbances from turbulence in the surrounding cocoon. These shocks are similar to reconfinement shocks and indeed involve regions of overpressure and underpressure in the jet. Transient annular shocks also occur in the inner parts of the jet backflow; these features 
are particularly frequent near the head of the jet.

In order to show the effect of orientation on the appearance of the lobe, we show, in Figure 4, a logarithmically scaled density image, together with synthetic radio images at orientations of $0^{\circ}-90^{\circ}$ at intervals of $15^{\circ}$. As shown in Saxton et al. (2002) jets rendered at orientations almost in the plane of the sky, $70^{\circ} \lesssim \theta \lesssim 90^{\circ}$, may show round hot-spots and knots on the jet axis, as well as more spatially extended and transient shock features that appear almost linear in projection. These do not resemble the arcs of Hercules A. Neither do we find morphologies resembling Hercules $\mathrm{A}$ when jets are rendered at a small angle to the line of sight, $\theta \lesssim 30^{\circ}$ : in such cases the extended shock features appear in projection as a clutter of tightly overlapping circles and knots. We find the best resemblance in frames rendered at $\theta \approx 45^{\circ}$.

Examples from simulations with a range of jet parameters $(\eta, M)$ are $\operatorname{shown}^{3}$ in Figures 5-8. These are discussed further below.

\subsection{Entrainment}

As detailed in Saxton et al. (2002), the hot-spot vanishes and reforms in an irregular cycle in which the jet is pinched off and temporarily obstructed by turbulence and/or the entrainment of dense gas in the cocoon, followed by the jet burrowing forward again. The effect of turbulence was documented in Saxton et al. (2002). This surging is most extensive and frequent for jets with low $\eta$. In the present paper, we also show in Figure 3, the effect of entrainment. Dense gas is swept into the cocoon and directly impedes the jet. This effect is particularly prominent at low Mach numbers, as one would expect from the Mach number dependence of the classical Kelvin-Helmholtz instability. In the density images in the figure we show a time series of snapshots that show dense (white) fingers of gas entrained into the lobe and partially, then totally, obstructing the passage of an $(\eta, M)=\left(10^{-4}, 2\right)$ jet. For a time, the extreme end of the lobe is being starved of jet plasma and consequently does not show a hot-spot.

In a significant fraction of instants in each of our simulations of light jets, the hot-spot is temporarily absent or dimmer than one or more brighter shock structures in the backflow, such as the annular rings. This occurs approximately $20 \%-50 \%$ of the time at all Mach numbers. To quantify the location of the hot-spot, we show in Figure 2 the trends in the

\footnotetext{
${ }^{3}$ Animations of the ray-traced frames from all our choices of the system parameters are presented at http://maker.anu.edu.au/radiojets/herculesa/.
} 
cumulative distributions of the ratios of the hot-spot to bow shock locations. That is, the plots show the probability that the ratio of hot-spot distance to bow-shock distance is less than a certain value. Note that the probability of a embedded hot-spot is greater at $M=2$ and also, for a given Mach number, at lower $\eta$.

\subsection{Dependence on the Mach number and density ratio}

Let us discuss some of the features of the simulations and in particular their dependence on Mach number and density ratio. The jet Mach number has a substantial effect on the typical brightness contrast between the background radio lobe and the shock features near the head of the jet. As expected on general theoretical grounds, and as shown in our previous studies (Saxton et al. 2002), for a given value of $\eta$ the cases with a larger jet Mach number show a greater brightness contrast. As $M$ increases, the ray-traced intensity maps are increasingly dominated by the transient frontal shock features near the head of the jet: a bright hot-spot comprising the jet termination shock, one of the nearer biconical shocks within the jet, or else a singularly bright annular shock emerging from the division of an older hot-spot. The background emission of the cocoon and secondary rings at lower $z$ positions, are relatively faint.

\subsection{Extracts from the simulations}

It is not feasible to show all of the frames from all of the simulations that we have carried out. In this subsection we show a small sample that is designed to illustrate the trends with Mach number and density ratio that we have noticed.

Snapshots from an $(\eta, M)=\left(10^{-4}, 50\right)$ simulation that exhibit ring structure are shown in Figure 5. Although approximately $3 \%-10 \%$ of the frames from the entire simulation are qualitatively similar to the multi-ringed morphology of Hercules A, the brightness contrast is too great. Where there appears a prominent ring, it is usually a lone product of a hot-spot's temporary disruption, and there are no other features of comparable brightness.

Simulations with lower Mach number produce a multiple ring morphology typically with a lower brightness contrast than for higher Mach numbers. In Figures 6 and 7 we show

snapshots of the $(\eta, M)=\left(10^{-4}, 5\right)$ and $(\eta, M)=\left(10^{-2}, 5\right)$ simulations that again illustrate the production of multiple rings of comparable brightness, separated appreciably along the length of the jet. The rings occurring furthest behind the head of the jet often appear in proximity to diamond shocks in the jet. Approximately $7 \%-15 \%$ of the $M=5$ images 
are qualitatively similar to Hercules A. The contrast between the bright features in the lobe compared to the rest of the lobe is less than for $M=50$. Nevertheless, note that the $\eta=10^{-2}$ images display a larger contrast than $\eta=10^{-4}$. This is a definite trend that is also evident in the intermediate $\eta=10^{-3}$ images (not shown).

In the $(\eta, M)=\left(10^{-4}, 2\right)$ simulation (Figure 8) the rings have a brightness contrast relative to the cocoon that is less than or equal to the observed factor $(\sim 3)$. Compared to the hot-spots in the simulations with higher $M$, the brightest point on the jet axis is typically quite far to the left of the brightest rings. In $M=2$ simulations, the cocoon and hot-spot together are dim enough that the jet itself appears prominent in comparison. This reduced contrast is obvious in all $M=2$ simulations. The dependence on $\eta$ is not as marked as it is for higher Mach numbers.

\section{Discussion and Conclusions}

\subsection{Morphology}

Our simulations show that a jet with constant fluxes of mass, momentum and energy may nonetheless vary dramatically in its morphology and extent within a jet dynamical time scale, $t_{\mathrm{dyn}} \equiv 2 r_{\mathrm{j}} / c_{\mathrm{s}, \mathrm{j}}$. In extremely light, low Mach number simulations, e.g. with $(\eta, M)=$ $\left(10^{-4}, 2\right)$, turbulence and dense obstructions in the backflow are sufficiently important that they can pinch and break the jet essentially anywhere along its length. The disconnected forward parts of the jet are then rapidly mixed into the rest of the cocoon. A new hotspot occurs at the new, recessed, jet terminus and this hot-spot gradually burrows forward through the cocoon until it reaches the forward working surface of the lobe once more. In the transitions of these extreme surging events, arrays of ring-like shocks, derived from the disconnected jet plasma, often exceed in brightness the hot-spots and diamond shocks within the jet. This is our explanation for the rings of enhanced radio intensity in the western lobe of Hercules A.

The surging jet dynamics revealed in simulations puts the western radio emitting structures into a new perspective. If we overlook the distraction of the rings and outer parts of the western radio lobe, the innermost "shell" identified in Mason et al. (1988) and Morrison \& Sadun (1996) actually resembles a hot-spot in a traditional FR 2 radio source. This is located at approximately $46 \%$ of the distance from the nucleus to the outer edge of the lobe. This may be the present western hot-spot, and the larger, outer western radio lobe may be the product of an earlier stage when the jet was not disrupted and reached out to a larger distance. 
The apparent absence of hot-spots near the outer edges of the radio lobes of Hercules A is a distinctive feature reproduced in many instants in all of our simulations. However, this phenomenon is most dramatic at $M=2$. Moreover, the occurrence of recessed hot-spots in the $M=2$ simulations is greatest for $\eta=10^{-4}$. The probability of an $(\eta, M)=\left(10^{-4}, 2\right)$ jet showing a jet terminus located at less than $50 \%$ of the distance to the outer edge of the lobe, is approximately 0.4 .

The differences between the $M=2$ and $M \geq 5$ simulations probably lies in the effective entrainment of matter at lower Mach numbers that is reflected in the higher rate of growth of unstable modes for low $M$. The minor dependence on $M \geq 5$ of the cumulative distribution functions shown in Figure 2 may be due to the increasingly vigorous nature of turbulent eddies in the cocoon. For these Mach numbers, as well, the lower $\eta$ jets are more prone to disruption.

The notion of a low Mach number in Hercules A is also consistent with the morphology of the eastern jet, since both jets presumably have similar properties when they emerge from the nucleus. However, inherent instabilities may cause differences of appearance at any particular moment on arcminute scales. The long "helical" portion of the eastern jet (upper panel of Figure 1) has the appearance of a jet that has become unstable, that is possibly decelerating to a transonic velocity and, as a result, becoming turbulent. The transition of a relativistic jet to turbulent transonic flow occurs when $\rho_{\mathrm{j}} c^{2} \sim 4 p$ (Bicknell 1995), which implies $\eta=\eta_{\text {crit }} \approx 4 \mathrm{k} T / \mu m_{\mathrm{p}} c^{2} \sim 10^{-4}$. For jets that have not made the transition to turbulent flow, $\eta<\eta_{\text {crit }}$. If the eastern jet is indeed transonic at the base of the eastern lobe, then both jets may emerge from the galaxy with a low Mach number, perhaps $2 \lesssim M \lesssim 5$. Since the western jet does not appear to have become fully turbulent, $\eta<10^{-4}$ confirming our preference for parameters for the Western jets that involve both a low Mach number and density ratio.

The brightness asymmetry of the prominent outer ring in the western lobe is an important characteristic that is reproduced in many bright shocked rings appearing in our simulations. This effect occurs because some shock-rings are ribbon-like, i.e. flat in crosssection, with the rings tilted in the $r-z$ plane. If the greater length of the cross-section is not exactly parallel to the $z$ axis then one side of the ring presents the observer with a wider projected area than the opposite side, at a particular orientation. The side with the narrower projected area is more extended along the line of sight, and therefore appears brighter than the side that is viewed at a less grazing angle. Therefore simple orientation effects are sufficient to explain the ring morphology of Hercules A, without resort to radical departures from axisymmetry. Nor is it necessary to consider the possibilities of directed Doppler boosting of emission from shocked plasma moving at highly relativistic velocities 
within the cocoon (c.f. discussion of the hot-spot of Pictor A in Saxton et al. 2002).

The relative sizes of the rings also naturally occurs as a result of the flow dynamics described here. As Figures 5-8 show, ring shocks appear with different relative sizes in different instances. In particular, the smallest "ring" in the observations corresponds to the structure surrounding a recessed hot-spot.

\subsection{Radio brightness contrast}

The high-resolution radio intensity maps of Hercules A (Dreher \& Feigelson 1984; Gizani et al. 2002) indicate that the brightest parts of the rings are on the order of $\sim 2-5$ times the intensity of the radio lobe background. The flatter radio spectra of the rings indicates that they are indeed shock features like those that we have modeled in simulations. Note however, that the ring spectra are not as flat as what we expect from shocked plasma. This may indicate that they are relatively long-lived structures (in terms of synchrotron timescales) and this is also consistent with the rings lasting longer in the lower Mach number simulations. However, assessment of this possibility must await the publication of more detailed surface brightness and spectral index observations.

If the bright part of the ring is the thinnest projected area of the underlying ribbon-like annular shock, then by inspection, the outer ring has a thickness $\gtrsim 0.08$ times the diameter of the radio lobe in that vicinity. The surface brightness enhancement of the ring over the lobe $\sim 2.5$; hence the volumetric emissivities of material in average conditions within the ring and within the lobe generally has a ratio $j_{\nu \text {,ring }} / j_{\nu \text {,lobe }} \lesssim 31$. This contrast suggests shocks of a certain strength, since $j_{\nu} \propto \varphi p^{(3+\alpha) / 2}$ where $\alpha$ is the radio spectral index. The shock pressure increases by a factor $\sim M^{2}$. This implies that the Mach number of the ring shocks is $\sim 3$. Our simulations with $M=2,5$ are the best matches for this strength of shocks in the cocoon. This conclusion is constrained by the assumptions of the emissivity model (see $\S 3)$; however it is probably indicative.

Inspection of the synthetic radio images shows that in cases with $M=10$ or $M=50$, the contrast between the rings and the cocoon is typically much greater than the observationally required value $\sim 3$. Many of the morphologically selected instances from the $M=2$ and $M=5$ simulations show the appropriate contrast. For each choice of $M$, the densest jets, $\eta=10^{-2}$ produce more frames showing excessive contrast in the shock features near the terminus of the jet, relative to the secondary rings. Cases with $\eta=10^{-4}$ give the more frames with the desired contrast relationship between rings, and we expect this to be so for a real jet that is lighter than the parameter regime our calculations could attain, $\eta \ll 10^{-4}$. 
Thus, on this basis, the simulations reinforce the suggestion that the jets of Hercules A are very light and have Mach number in the range $2 \lesssim M \lesssim 5$.

\subsection{Orientation of Hercules A}

The spherical shell models of Mason et al. (1988) and Morrison \& Sadun (1996) imply geometric constraints on the orientation of the trail of shells. Assuming that such a model is correct, the shells probably are not in physical contact, otherwise their spherical form and brightness distribution would be affected greatly. If consecutive shells of radius $r_{n}$ and $r_{n+1}$ do not intersect in three dimensions, and yet are partially superimposed in projection on the sky, then they are aligned on a line at an inclination $\theta$, which is constrained by the inequality

$$
\sin \theta<\frac{\Delta z_{n, n+1}^{\prime}}{r_{n}+r_{n+1}}
$$

where $\Delta z_{n, n+1}^{\prime}=z_{n+1}^{\prime}-z_{n}^{\prime}$ is the separation of the shell origins projected onto the plane of the sky. For Hercules A, the constraints are $\theta<46^{\circ}, 47^{\circ}, 23^{\circ}$ for the pairs of visually overlapping rings (assumed to be circular) as shown in the Figures 2(a) and 3(a) of Morrison \& Sadun (1996).

However in our model, the luminous radio-emitting rings may in principle be separated by distances much less than their radii, and larger values of $\theta$ are feasible. On the other hand, our model explains the non-circularity of the rings, implying that $\cos \theta \approx b^{\prime} / a^{\prime}$ where $a^{\prime}$ and $b^{\prime}$ are the semi-major and semi-minor axes of the elliptical projection of a circular ring onto the plane of the sky. Apart from the innermost bright feature which we re-identify as a buried hot-spot or reconfinement shock, we distinguish up to five rings clearly in the radio images, (lower panel of Figure 1) from east to west: a partially filled ellipse, one dimmer partial elliptical arc, a bright nearly filled ellipse, a relatively dim ring and then the bright half-ring. In the lower panel of Figure 1 we exhibit the elliptical fits to some of the observed features, and we infer inclinations of $\theta \approx 45^{\circ} \pm 10^{\circ}$. These fits are demonstrative but not unique; the thickness of the radio structures allows some freedom in fitting ellipses as does the inclusion or exclusion of other faint filamentary features near each ring. However the fitted ellipses of projected ring-shocks, with their projected major axes approximately locally perpendicular to the jet are consistent with our model. 


\subsection{Implications for the cluster environment}

The two temperatures of diffuse X-ray emission from the cluster medium surrounding Hercules A may reflect the different states of gas upstream and downstream of the bow shock driven by the outer radio lobes. The observed temperature ratio is $1.7 \lesssim T / T_{\text {ism }} \lesssim 2.1$, which is comparable to the temperature ratios of the shocked thermal gas in some of our simulations. In Table 2 we show temperature ratios found at the point of the bow shock, and on the sides of the bow shock parallel to the jet axis. The tabulated temperature ratios indicate a lower Mach number $\sim 5$, if this explanation for the two-temperature structure of the local cluster medium is correct. The temperature ratios are too small $(\approx 1)$ for the $M=2$ simulations.

\subsection{Closing remarks}

We have presented a model for the western lobe of Hercules A in which the ring structures are explained as shocks in the jet cocoon, with a ribbon-like, approximately annular geometry. The absence of a strong hot-spot at the extremity of the radio lobe may be attributed to the surging of the head of the jet on length scales of $\sim 10^{2} \mathrm{kpc}$, as a result of the dynamics of the turbulent and entraining backflows in the radio lobe. The lack of a hot-spot near the edge of the lobe, the low contrast of the radio rings and the appearance of the Eastern jet all suggest a low Mach number $M \sim 2-5$, low density ratio $\eta<10^{-4}$ jet. In order that the jet power be consistent with the luminosity of the source, $\eta$ may be as low as $10^{-6}$ and this is consistent with our view of radio galaxy jets as being composed of relativistic plasma. We have suggested that the recessed hot-spot may be associated with one of the bright knots some $35^{\prime \prime}$ west of the core. Another possibility is that this knot is associated with a reconfinement shock and the hot-spot could well be absent. However, the fraction of the time when a hot-spot is entirely absent in these simulations is small.

Another appealing aspect of our modelling is that we have estimated an orientation such that the eastern jet makes an acute angle to the line of sight, $30^{\circ} \lesssim \theta<70^{\circ}$, consistent with the value $\theta \approx 50^{\circ}$ determined from the radio depolarization measurements of Gizani \& Leahy (1999).

With the simulations that we have carried only three out of the twelve have an energy flux that is close to the range of $\sim 2 \times 10^{45-46} \mathrm{erg} \mathrm{s}^{-1}$ identified in $\S 2$. However, the other nine simulations are useful in that their morphologies and contrasts serve to identify a trend towards the low Mach numbers and density ratios that is consistent with the region of parameter space defined by the energy budget. 
The notion of a reasonably low Mach number for the western jet in this source is compatible with the appearance of both sides. Both jets seems to be on the verge of a transition to full turbulence. It is noteworthy that this may be occurring in a source which is so much more powerful than borderline FR 1/FR 2 sources. The production of turbulent jets in Hercules A is most likely the result of the large core-radius of the hot X-ray emitting gas that gradually decelerates the initially relativistic jets to a low Mach number. Normally one expects the X-ray core-radius to be of the order of the optical core radius since at small radii the matter distribution is dominated by the optical potential. At present we can only speculate that the large X-ray core radius in Hercules A is related to the merger apparent in the optical observations. (Minkowski 1957; Sadun \& Hayes 1993).

Finally we should comment on the relevance of two-dimensional simulations. These are justified a priori on the basis of observed ring-like structure and the simulations seem to capture a lot of the physics that is relevant to the observations. Nevertheless, it would be desirable to carry out three-dimensional simulations and test some of the features of the present work. For example, how much of a perturbation from axi-symmetry is allowable before the ring structure is destroyed and how sensitive is the Mach number dependence of the entrainment effects to the dimensionality of the simulations?

\section{Acknowledgments}

This work was supported by an Australian Research Council Large Grant, A69905341 and grants of computing time from the ANU Supercomputer Facility. We are grateful to Drs N. Gizani, M. Garrett and P. Leahy for permission to publish their radio image (Figure 1). 


\section{REFERENCES}

Allington-Smith, J. R., Ellis, R., Zirbel, E. L., \& Oemler, A. J. 1993, ApJ, 404, 521

Bicknell, G. V. 1994, Ap. J., 422, 542

Bicknell, G. V. 1995, ApJS, 101, 29

Bicknell, G. V. 2000 Elsevier, in press

Blondin, J. M. \& Lukfin, E. A. 1993, ApJS, 88, 589

Carvalho, J. C. \& O’Dea, C. P. 2001, ApJS, accepted, -

Colella, P. \& Woodward, P. R. 1984, J. Comput. Phys., 54, 174

Conner, S. R., et al. 1998, AJ, 115, 37

Dreher, J. W. \& Feigelson, E. D. 1984, Nature, 308, 43

Fanaroff, B. L. \& Riley, J. M. 1974, MNRAS, 167, 31P

Garrington, S. T, Leahy, J. P., Conway, R. G., \& Laing, R. A. 1988, Nature, 331, 147

Gizani, N., Garrett, M. A., \& Leahy, J. P. 2000, in EVN Symposium 2000, Proceedings of the 5th european VLBI Network Symposium held at Chalmers University of Technology, Gothenburg, Sweden, June 29 - July 1, 2000, Eds.: J.E. Conway, A.G. Polatidis, R.S. Booth and Y.M. Pihlström, published Onsala Space Observatory, p. 1919

Gizani, N. A. B., Garrett, M. A., \& Leahy, J. P. 2002, PASA, 19, 69

Gizani, N. A. B. \& Leahy, J. P. 1999, New Astronomy Review, 43, 639

Greenstein, J. L. 1962, ApJ, 135, 679

Kellermann, K. I., Pauliny-Toth, I. I. K., \& Williams, P. J. S. 1969, ApJ, 157, 1

Laing, R. A. 1988, Nature, 331, 149

Mason, A., Morrison, P., \& Sadun, A. C. 1988, Nature, 333, 640

Meier, D. L., Sadun, A. C., \& Lind, K. R. 1991, ApJ, 379, 141

Minkowski, R. 1957, in IAU Symp. 4: Radio astronomy, Volume 4107

Morrison, P. \& Sadun, A. 1996, MNRAS, 278, 265 
Owen, F. N. \& Laing, R. A. 1989, MNRAS, 238, 357

Perley, R. A., Bridle, A. H., Willis, A. G., \& Fomalont, E. B. 1980, AJ, 85, 499

Rosen, A., Hughes, P. A., Duncan, G. C., \& Hardee, P. E. 1999, ApJ, 516, 729

Sadun, A. C. \& Hayes, J. J. E. 1993, PASP, 105, 379

Saxton, C. J., Sutherland, R. S., Bicknell, G. V., Blanchet, G. F., \& Wagner, S. J. 2002, A\&A, submitted, -

Siebert, J., Kawai, N., \& Brinkmann, W. 1999, A\&A, 350, 25

Trussoni, E., Feretti, L., Massaglia, S., \& Parma, P. 2001, A\&A, 366, 788

van Breugel, W. \& Fomalont, E. B. 1984, ApJ, 282, L55

Yates, M. G., Miller, L., \& Peacock, J. A. 1989, MNRAS, 240, 129 

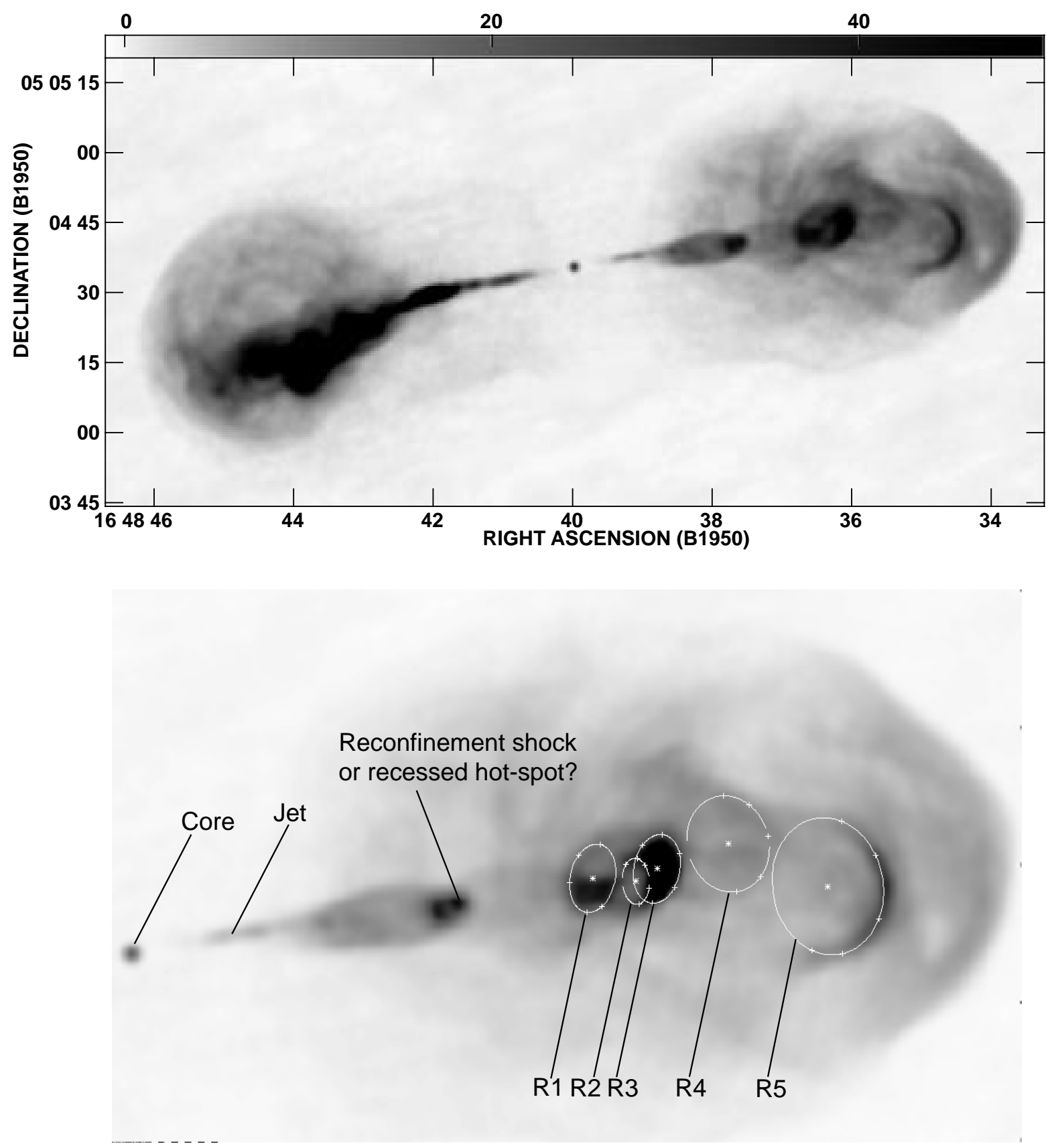

Fig. 1.- Radio image (at $18 \mathrm{~cm}$ ) of Hercules A, its lobes, jets and associated rings, from Gizani et al. (2002) and published with the permission of those authors. The lower panel is an enhanced-contrast sub-region showing the core, jet and shock features. Some apparent ring-like structures are marked $R 1-R 5$. The illustrative fitted ellipses are at inclinations $\theta=36^{\circ}, 32^{\circ}, 38^{\circ}, 50^{\circ}, 47^{\circ}$ respectively. 

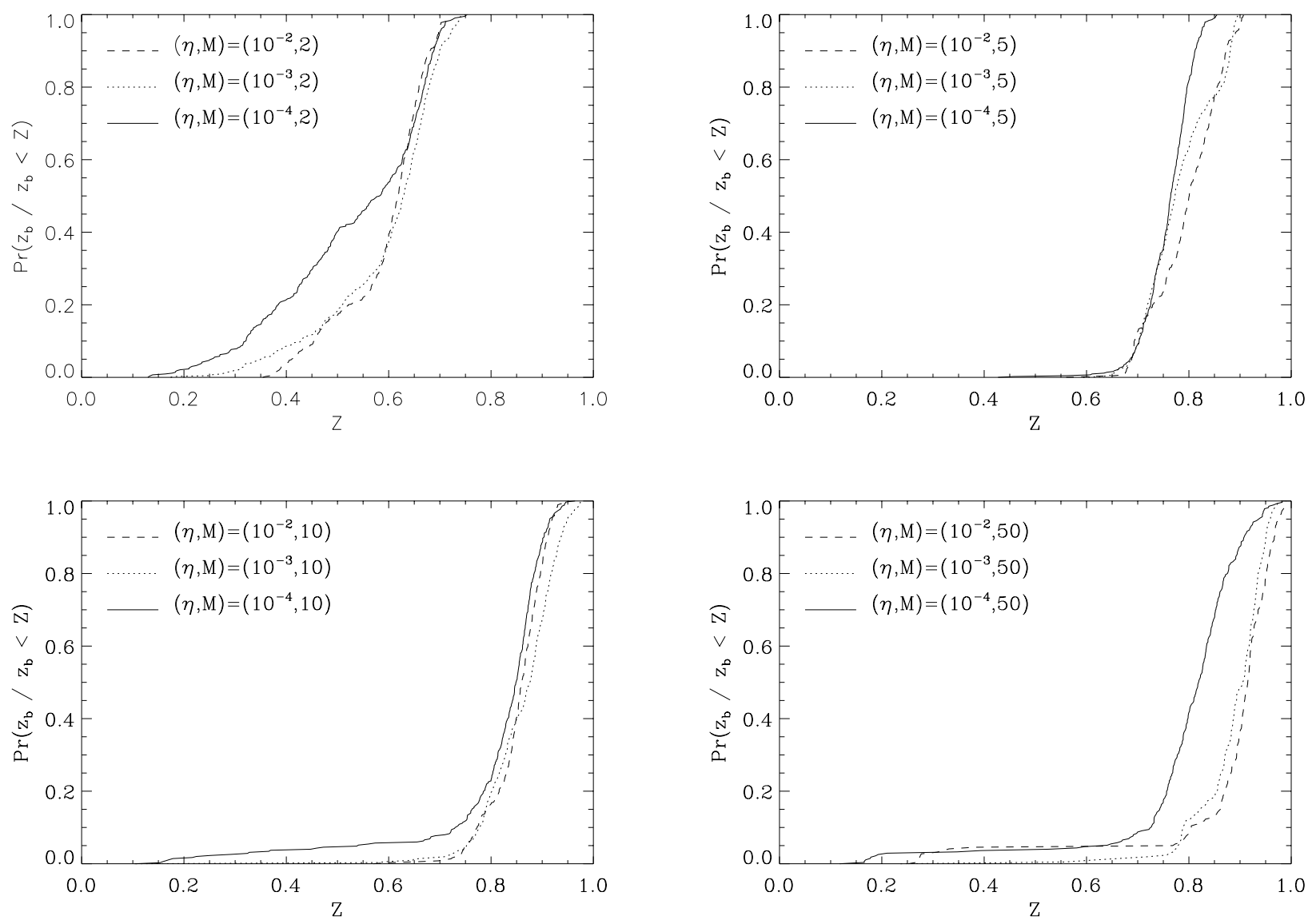

Fig. 2.- Cumulative distribution functions expressing the frequency and extent of surging in terms of ratios of the $z$ positions of the bow shock and jet head. The jet's head position $z_{\mathrm{h}}$ is defined as the forwardmost cell where the jet plasma remains unmixed with external gas, i.e. $\varphi=1$. We show cases with $M=2,5,10,50$. These are simulations with an open left boundary, and $\eta=10^{-2}, 10^{-3}, 10^{-4}$ for dashed, dotted and solid lines respectively. 

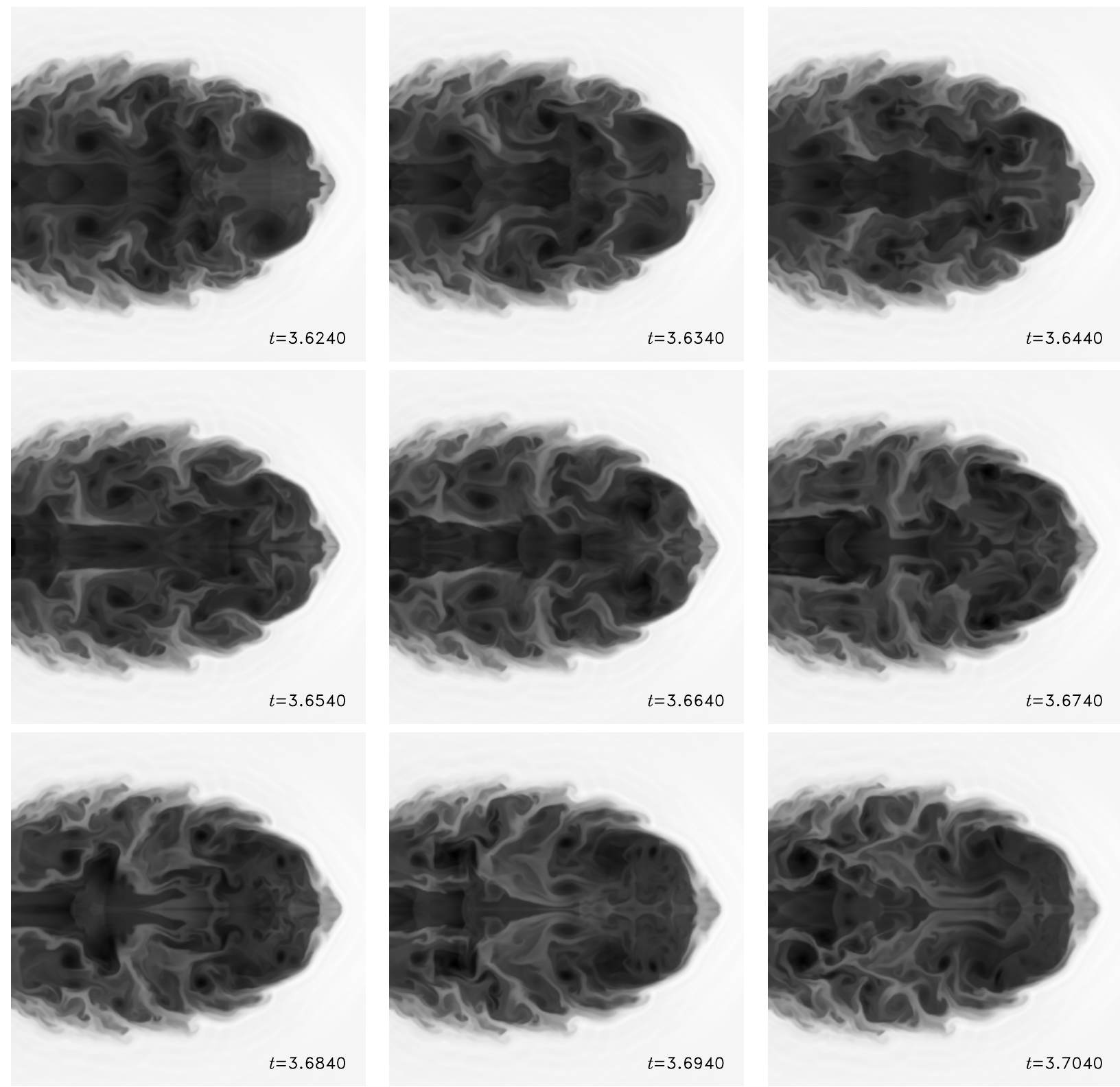

Fig. 3.- A sequences of $\log \rho$ cross-sections of the jet showing as it is affected by dense fingers of external gas entrained into the cocoon. In this sequence, $(\eta, M)=\left(10^{-4}, 2\right)$, the dark color represents the low density jet material; the lightest areas represent the dense ambient gas. 

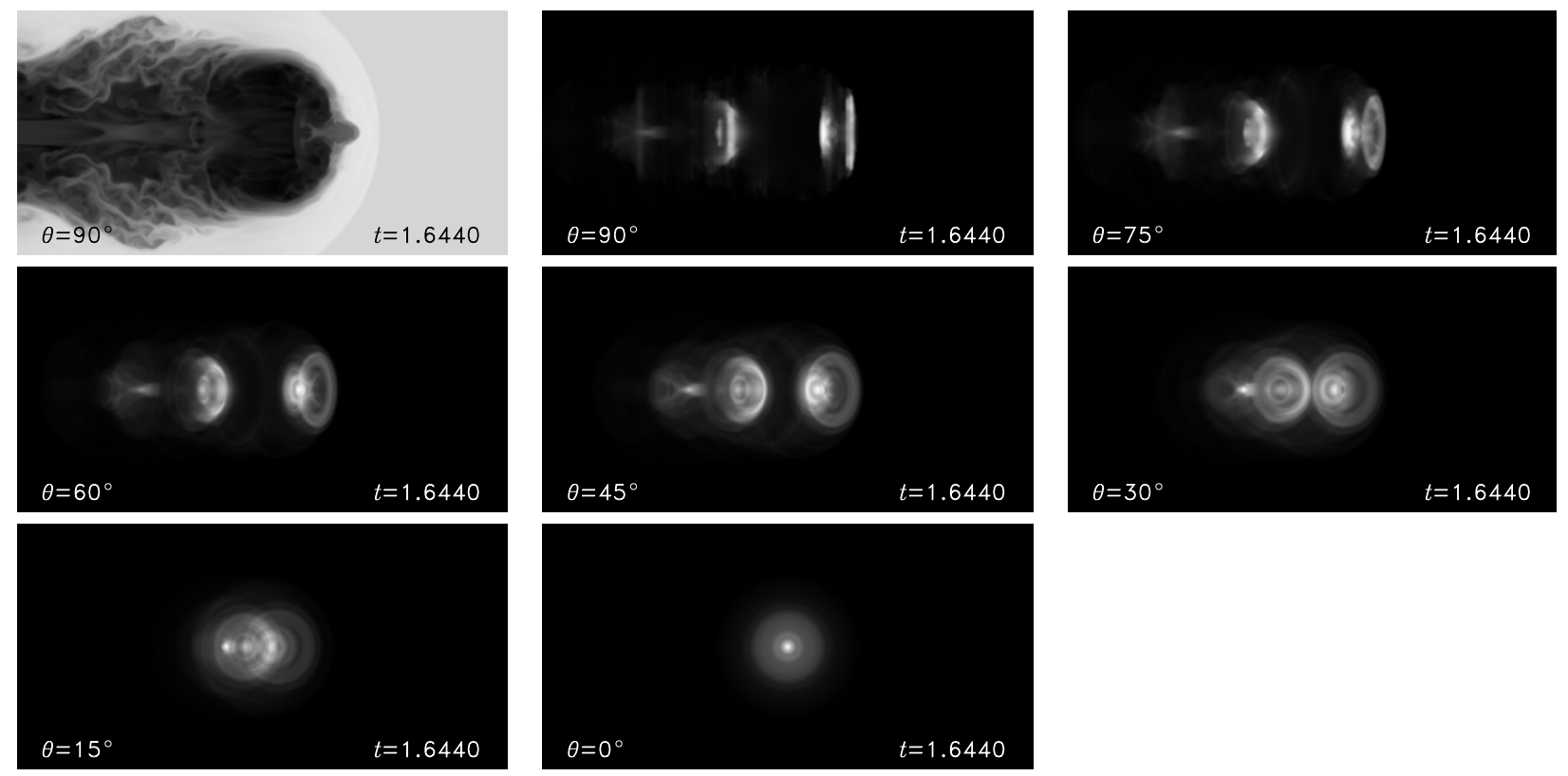

Fig. 4.- A sub-region of the $(\eta, M)=\left(10^{-4}, 5\right)$ simulation, showing $\log \rho$ (top left panel), and a corresponding sequence of simulated intensity maps for different orientations, with an angle $\theta$ between the jet axis and the line of sight. 

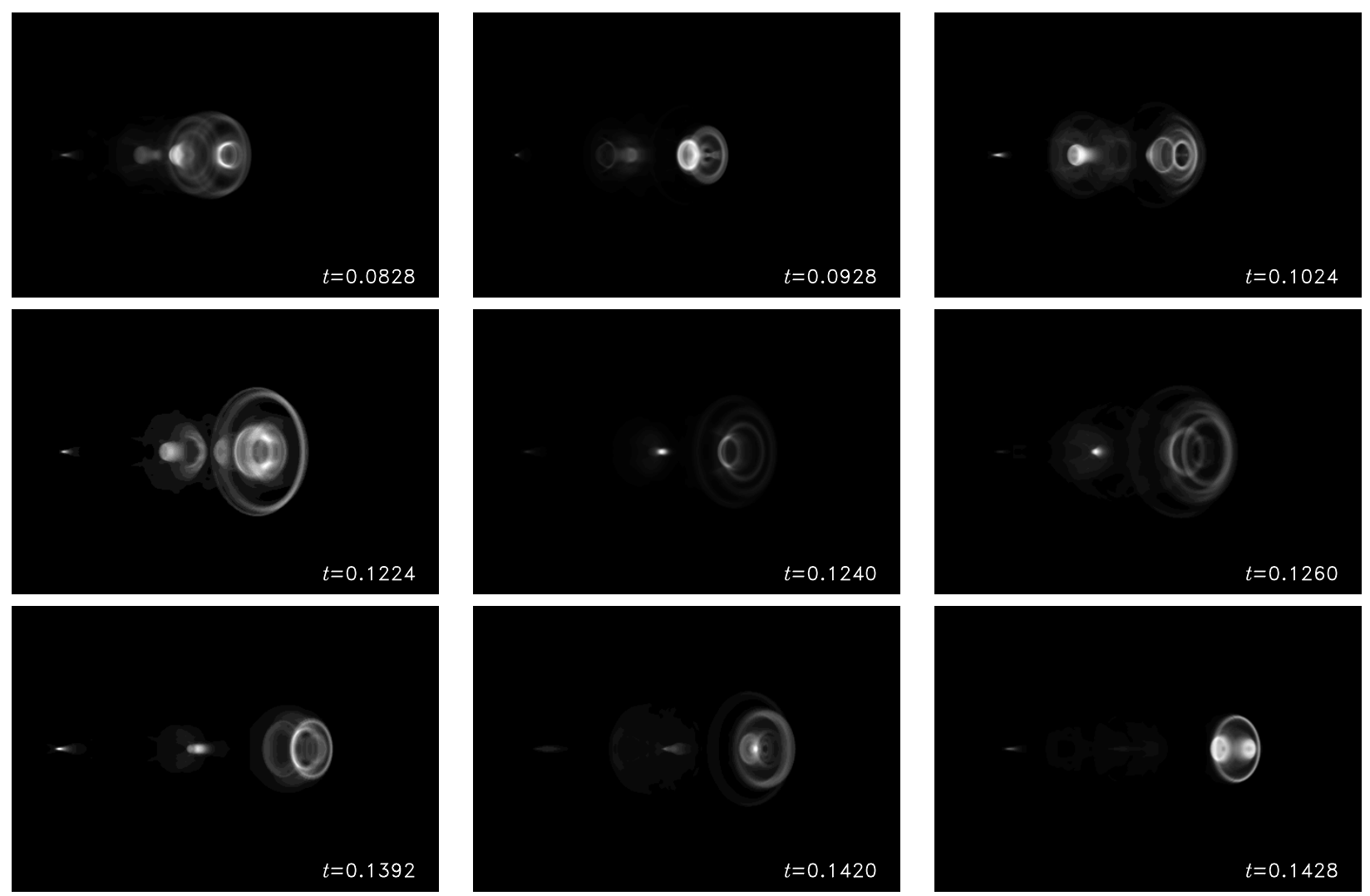

Fig. 5.- Selected images rendered with the jet at an orientation of $\theta=45^{\circ}$ to the line of sight, from the simulation with jet parameters $(\eta, M)=\left(10^{-4}, 50\right)$ and an open left boundary. This figure shows the high brightness contrast of the rings and hot-spot with respect to the rest of the lobe when the Mach number is high. 

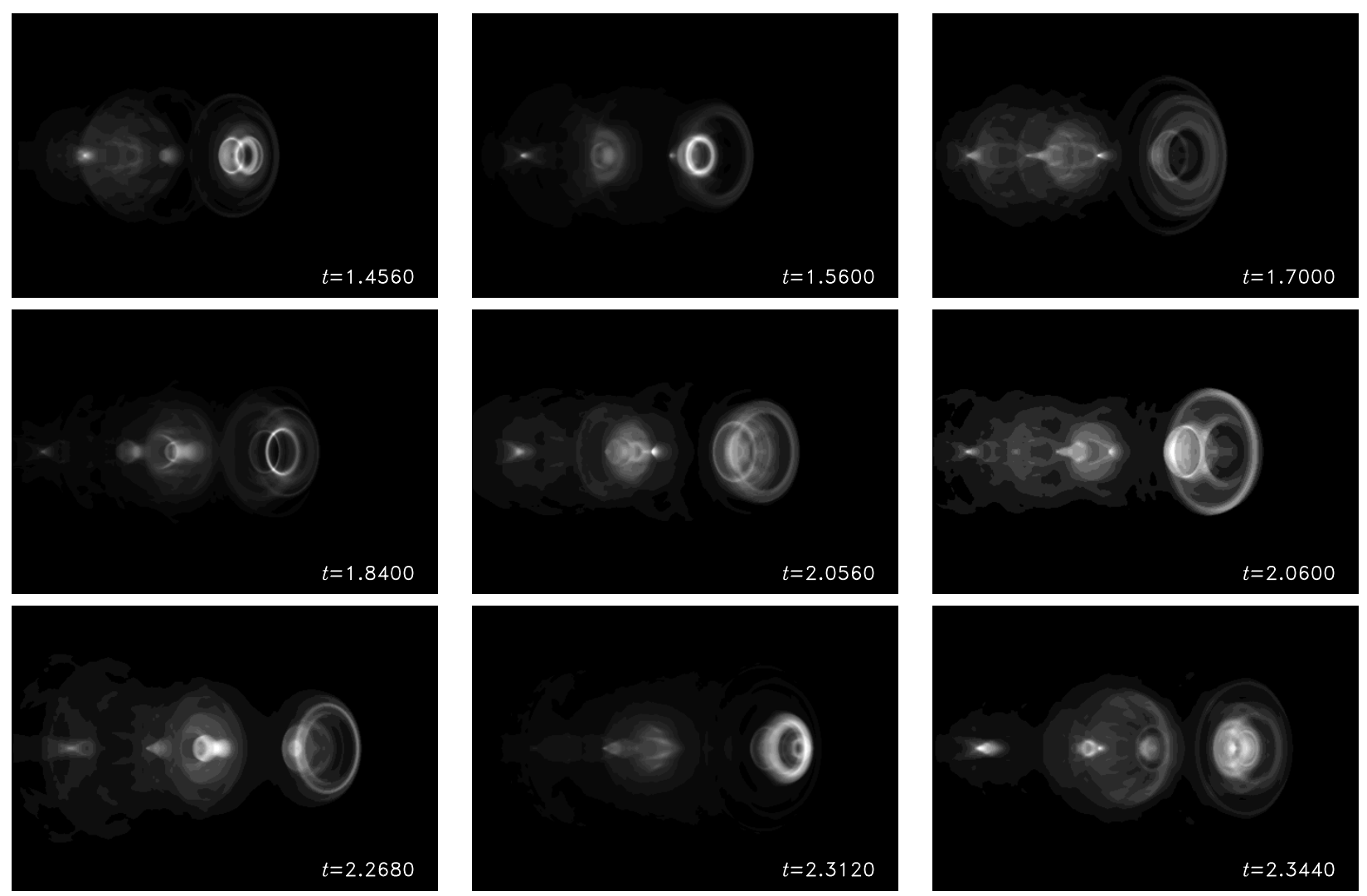

Fig. 6. - Selected images rendered with the jet at an orientation of $\theta=45^{\circ}$ to the line of sight, from the simulation with jet parameters $(\eta, M)=\left(10^{-4}, 5\right)$ and an open left boundary. The contrast of the rings and hot-spots against the radio lobe is less marked than for $(\eta, M)=$ $\left(10^{-4}, 50\right)$. 

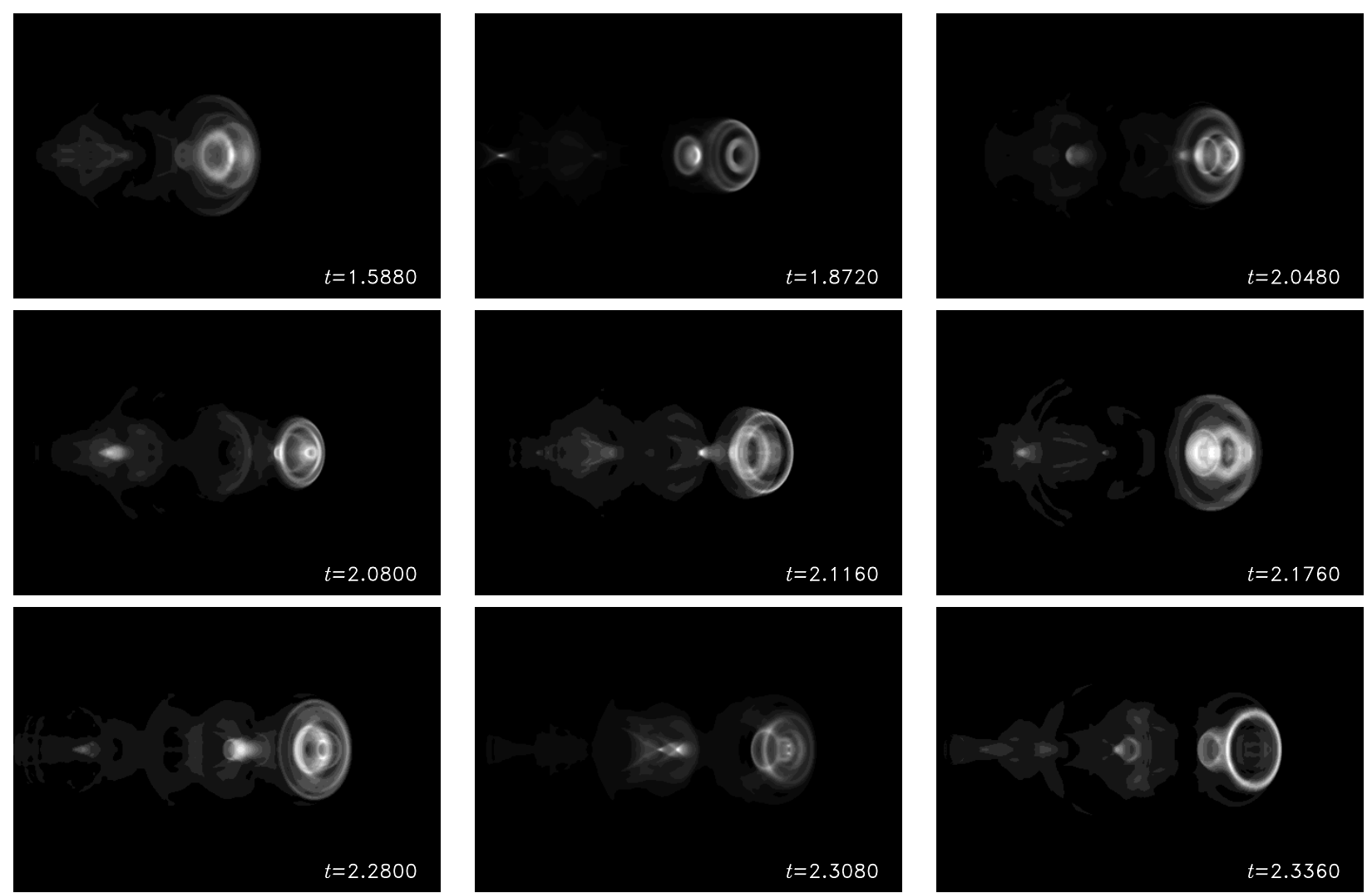

Fig. 7.- Selected images rendered with the jet at an orientation of $\theta=45^{\circ}$ to the line of sight, from the simulation with jet parameters $(\eta, M)=\left(10^{-2}, 5\right)$ and an open left boundary. Compared to $(\eta, M)=\left(10^{-4}, 5\right)$, this sequence shows a greater concentration of bright features towards the head of the lobe. There are few instances of equally bright well-separated rings. 

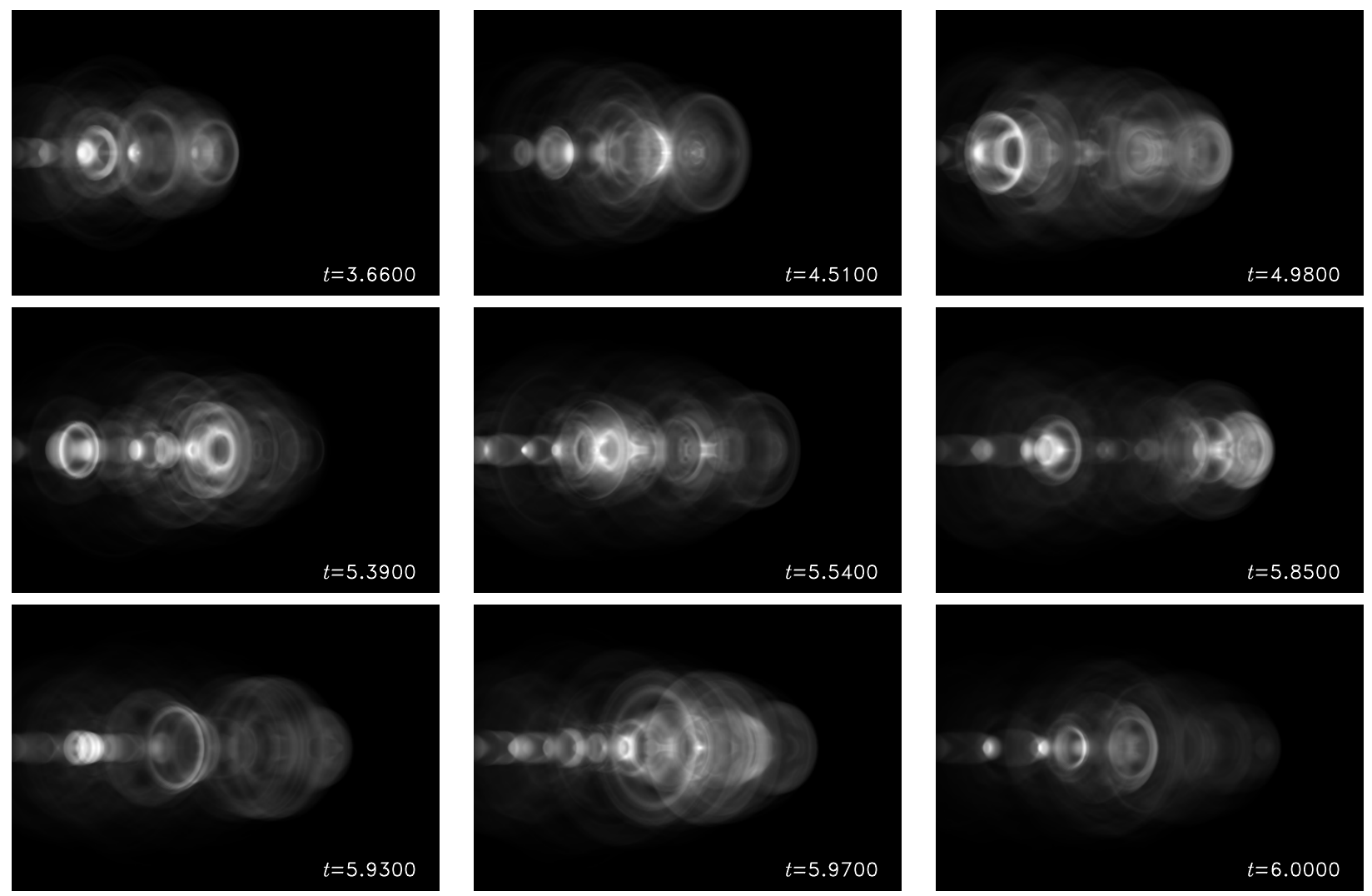

Fig. 8. - Selected images rendered with the jet at an orientation of $\theta=45^{\circ}$, from the simulation with jet parameters $(\eta, M)=\left(10^{-4}, 2\right)$ and an open left boundary. The lower brightness contrast of the rings, jet knots and hot-spots with respect to the rest of the lobe, compared to the higher density, higher Mach number simulations, is apparent. 
Table 1: Classical evaluations of the velocity, mass flux, force and power of a jet with radius $r_{\text {jet }}=0.5 \mathrm{kpc}$ for our choices of the parameters $(\eta, M)$. These are non-relativistic evaluations, assuming cosmic abundances, an electron number density of $n_{0}=7.8 \times 10^{-3} \mathrm{~cm}^{-3}$ and a temperature of $k T_{\mathrm{ism}}=2.45 \mathrm{keV}$ in the undisturbed ambient medium.

\begin{tabular}{|c|c|c|c|c|c|}
\hline$\eta$ & M & $\begin{array}{l}\frac{v_{\mathrm{j}}}{\left(T / T_{\mathrm{ism}}\right)^{1 / 2}} \\
\left(\mathrm{~cm} \cdot \mathrm{s}^{-1}\right)\end{array}$ & 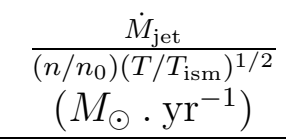 & $\begin{array}{c}\frac{F_{\text {jet }}}{\left(n / n_{0}\right)\left(T / T_{\text {ism }}\right)} \\
(\text { dyn })\end{array}$ & $\begin{array}{c}\frac{L_{\text {jet }}}{\left(n / n_{0}\right)\left(T / T_{\text {ism }}\right)^{3 / 2}} \\
\left(\mathrm{erg} \cdot \mathrm{s}^{-1}\right)\end{array}$ \\
\hline $10^{-2}$ & 2 & $1.59 \times 10^{9}$ & $2.87 \times 10^{-2}$ & $3.31 \times 10^{33}$ & $4.00 \times 10^{42}$ \\
\hline $10^{-3}$ & 2 & $5.03 \times 10^{9}$ & $9.08 \times 10^{-3}$ & $3.31 \times 10^{33}$ & $1.26 \times 10^{43}$ \\
\hline $10^{-4}$ & 2 & $1.59 \times 10^{10}$ & $2.87 \times 10^{-3}$ & $3.31 \times 10^{33}$ & $4.00 \times 10^{43}$ \\
\hline $10^{-2}$ & 5 & $3.97 \times 10^{9}$ & $7.18 \times 10^{-2}$ & $1.84 \times 10^{34}$ & $4.00 \times 10^{43}$ \\
\hline $10^{-3}$ & 5 & $1.26 \times 10^{10}$ & $2.27 \times 10^{-2}$ & $1.84 \times 10^{34}$ & $1.26 \times 10^{44}$ \\
\hline $10^{-4}$ & 5 & $3.97 \times 10^{10}$ & $7.18 \times 10^{-3}$ & $1.84 \times 10^{34}$ & $4.00 \times 10^{44}$ \\
\hline $10^{-2}$ & 10 & $7.95 \times 10^{9}$ & $1.44 \times 10^{-1}$ & $7.23 \times 10^{34}$ & $2.94 \times 10^{44}$ \\
\hline $10^{-3}$ & 10 & $2.51 \times 10^{10}$ & $4.54 \times 10^{-2}$ & $7.23 \times 10^{34}$ & $9.30 \times 10^{44}$ \\
\hline $10^{-4}$ & 10 & $7.95 \times 10^{10}$ & $1.44 \times 10^{-2}$ & $7.23 \times 10^{34}$ & $2.94 \times 10^{45}$ \\
\hline $10^{-2}$ & 50 & $3.97 \times 10^{10}$ & $7.18 \times 10^{-1}$ & $1.80 \times 10^{36}$ & $3.57 \times 10^{46}$ \\
\hline $10^{-3}$ & 50 & $1.26 \times 10^{10}$ & $2.27 \times 10^{-1}$ & $1.80 \times 10^{36}$ & $1.13 \times 10^{47}$ \\
\hline $10^{-4}$ & 50 & $3.97 \times 10^{11}$ & $7.18 \times 10^{-2}$ & $1.80 \times 10^{36}$ & $3.57 \times 10^{47}$ \\
\hline
\end{tabular}

Table 2: Typical temperatures in shocked thermal gas at the front of the bow shock $\left(T_{\mathrm{b}} / T_{\text {ism }}\right)$ and on the flank where the shock is approximately parallel to the jet $\left(T_{\mathrm{f}} / T_{\mathrm{ism}}\right)$, for various choices of the system parameters.

\begin{tabular}{cccccccccc}
$\eta$ & $M$ & $T_{\mathrm{b}} / T_{\text {ism }}$ & $T_{\mathrm{f}} / T_{\text {ism }}$ & & $\eta$ & $M$ & $T_{\mathrm{b}} / T_{\text {ism }}$ & $T_{\mathrm{f}} / T_{\text {ism }}$ \\
\cline { 1 - 5 } $10^{-2}$ & 2 & 1.08 & 1.02 & & $10^{-2}$ & 10 & 3.28 & 1.54 \\
$10^{-3}$ & 2 & 1.08 & 1.02 & & $10^{-3}$ & 10 & 3.64 & 1.76 \\
$10^{-4}$ & 2 & 1.11 & 1.03 & & $10^{-4}$ & 10 & 2.97 & 1.45 \\
& & & & & & & & \\
$10^{-2}$ & 5 & 1.71 & 1.18 & & $10^{-2}$ & 50 & 20.6 & 4.45 \\
$10^{-3}$ & 5 & 1.63 & 1.13 & & $10^{-3}$ & 50 & 27.1 & 6.53 \\
$10^{-4}$ & 5 & 1.69 & 1.17 & & $10^{-4}$ & 50 & 13.9 & 6.71 \\
& & & & & & & \\
\hline
\end{tabular}

